\title{
Chi ha prodotto il logo per la Nefrologia Narrativa?
}

\section{Cari lettori,}

La locomotiva della Nefrologia Narrativa (NN) si è messa in moto... e chi la ferma piu? Poteva un simile progetto non avere un suo logo? Ovviamente no!

Dopo alcuni tentativi infruttuosi del sottoscritto, lo stesso ha gettato diverse "esche" sino a che non ha ricordato l'esistenza di Lorenzo Console, un giovane studente che sogna di scrivere e scrive di sogni... uno che dorme tanto, e per il quale è quindi semplice sognare. Un giovane che si appassiona per tutto ciò che è racconto e narrazione ma anche per le immagini, l'arte, il mondo del visivo da cui ha appreso l'arte della grafica pubblicitaria. Uno che come suo miglior marchio ha l'amore per le storie... quale miglior curriculum per porsi in lizza come inventore del logo per la NN del GTND?

Ha risposto immediatamente alle mie aspettative centrando appieno il mio sogno e non ho potuto che scegliere questo oramai "nostro" logo (Fig. 1), pur dovendo licenziare a malincuore altri pervenutimi assolutamente belli e significativi... ma questo soddisfaceva appieno le aspettative dell' Editor in Chief del Giornale... e così è andata.

La felicità e la soddisfazione che mi derivano da questo progetto di NN e da come sta avanzando questa locomotiva "a tutto vapore" mi inducono a presentarvi brevemente Lorenzo, al quale credo commissionerò di scrivere di "narrativa" su queste pagine prossimamente.

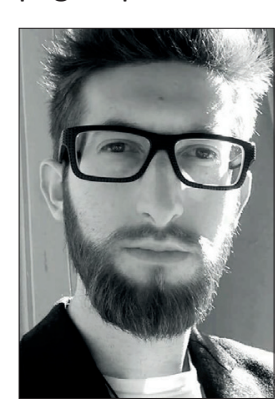

Lorenzo Console

Lorenzo Console nasce nella culla del Rinascimento, Firenze, il 23 febbraio 1994. Dopo aver conseguito il diploma nel 2013 presso l'Istituto Statale d'Arte come grafico pubblicitario, si trasferisce a Bologna per frequentare il DAMS con indirizzo Cinema e Arti Visive.

Laureatosi nel 2016 si sposta a Milano per proseguire i suoi studi di scrittura e poesia. Giovanissimo, inizia a scrivere e il suo talento trova riconoscimento nei concorsi "Il giardino di Babuk" (Roma, 2014) e "TuttoMondo Contest" (Roma, 2015 - finalista). Esordisce inoltre con diverse pubblicazioni: LeggiMi (eBook e racconto pubblicato in TuttaFirenze, 2013), Il Raccontario (LudovicaGreta Editore, 2014), D'Estro Artistico (Cellar Door, 2015). Ha collaborato inoltre con la compagnia teatrale VentiLucenti di Firenze e al cortometraggio Gran Finale di Valerio Groppa nel 2015. Nel 2016 arriva terzo classificato nella sezione microletteratura al Premio Fogazzaro, mentre nel 2017 entra nell'antologia Giovani Scrittori Iulm.

Marco Lombardi Editor in Chief Giornale di Tecniche Nefrologiche e Dialitiche 